\title{
Detection of SARS-CoV-2 infection by RT-PCR test: factors influencing interpretation of results
}

\author{
Kiran Munne ${ }^{1}$ (ID $\cdot$ Venkanna Bhanothu $^{2} \cdot$ Vikrant Bhor $^{3} \cdot$ Vainav Patel $^{4}$. \\ Smita D. Mahale ${ }^{5}$ Shailesh Pande ${ }^{2}$
}

Received: 7 October 2020/ Accepted: 23 April 2021/Published online: 3 May 2021

(C) Indian Virological Society 2021

\begin{abstract}
In this current pandemic of coronavirus disease 2019 (COVID-19), prompt interventions in terms of early detection and clinical management along with isolation of positive cases is of utmost importance. This helps to limit not only the spread of severe acute respiratory syndrome coronavirus 2 (SARS-CoV-2) infections but also the morbidity and mortality associated with it. Different strategies for screening of COVID-19 in containment zones and noncontainment areas include testing of symptomatic patients and their contacts in fever clinics, hospital-based testing, testing on demand and population-based screening. The choice of tests like reverse-transcription polymerase chain reaction (RT-PCR), rapid antigen testing (RAT) or antibody test depends upon these strategies and also the turnaround time. Currently, RT-PCR is considered the gold standard for COVID-19 detection. This commentary pro-
\end{abstract}

vides the insights and experiences on COVID-19 diagnosis by RT-PCR. The utility of this test is limited by several false positive, false negative and inconclusive results at early stages of infection, scarcity of reagents and lack of well-equipped labs including trained staff. Moreover, appropriate sample collection and transport, standard laboratory protocols, stringent quality control norms, good quality RNA extraction kits, PCR kits with suitable primers can help in improving accuracy of the test results. A careful assessment of clinical, radiological and molecular findings is required for identifying potential cases of COVID-19.

Keywords COVID-19 - Laboratory diagnosis · RT-PCR · SARS-CoV-2

2 Genetic Research Centre, ICMR-National Institute for Research in Reproductive Health, Jehangir Merwanji Street, Parel, Mumbai 400012, India

3 Molecular Immunology and Microbiology, ICMR-National Institute for Research in Reproductive Health, Jehangir Merwanji Street, Parel, Mumbai 400012, India

4 Department of Biochemistry, ICMR-National Institute for Research in Reproductive Health, Jehangir Merwanji Street, Parel, Mumbai 400012, India

5 ICMR-National Institute for Research in Reproductive Health, Jehangir Merwanji Street, Parel, Mumbai 400012, India 


\section{Introduction}

The most cost effective, reliable and considered to be the gold standard test for laboratory diagnosis of coronavirus disease 2019 (COVID-19) is quantitative fluorescence-real time reverse transcriptase polymerase chain reaction (RTPCR) $[2,3,14]$. It is reported that the sensitivity and specificity of RT-PCR is not $100 \%$. As per the published literature its sensitivity is estimated to be $70-98 \%$ and specificity is approximately 95\% [7, 13]. In addition, genetic diversity of the severe acute respiratory syndrome coronavirus-2 (SARS-CoV-2) plays an important role and may affect the results of the RT-PCR test $[9,10]$. Thus, it is very important to understand the limitations and pit falls of the technique. ICMR-NIRRH is an accredited testing centre for COVID-19 diagnosis and a centre for validation of related diagnostic reagents. We have tested and reported on approximately 74,000 samples during the period of April 2020-mid March 2021, using multiple diagnostic kits as well as different platforms. Herein, we provide our perspectives and suggestions that may help guide optimized testing for SARS-CoV-2 infection. The challenges encountered in the detection of COVID-19 by RT-PCR may be overcome by addressing the following issues.

\section{Selection of diagnostic test(s) and cognizance of clinical history}

It has been observed that patients with a strong clinical suspicion of COVID-19 with classical features may at times show negative results [5]. This could be because of primers used for the fragment of gene which may have mutations, amplification inhibitors, insufficient target (low copy number of virus in the patient) or sample degradation because of improper transport or handling of the samples $[3,9,10,14,15]$. Variability in the selection of RNA sequence for designing the primers used for detection of Viral RNA sequences may also affect results. Due to this, some approved (FDA and/or ICMR); kits can sometimes give inconclusive results. Thus, repeat testing of these inconclusive samples with approved RT-PCR kits having different target genes may be helpful. In such cases, interpretation of SARS-CoV-2 RT-PCR results should preferably be done after taking into consideration the clinical condition and the history of exposure $[12,15]$. A dialogue between the testing laboratory and the clinician can be an effective way for interpretation of the results and effective patient management $[12,15]$.

\section{Result interpretation}

Different commercial RT-PCR kits are currently available. Each kit differs with regards to specificity, and sensitivity thereby affecting overall outcome [1]. The kit with primers designed specifically for the novel Corona virus family and for most conserved regions of viral genomes (multiplexing/multiple target gene amplification) including strong internal controls should be preferred for COVID-19 diagnosis. This can help in reducing the false negative results. Still in some cases, mismatches between the primers and targeted regions are reported but the chances of such occurrences are very remote [8]. Validity of the assay can be improved by adding internal controls before nucleic acid extraction (extraction control) or before amplification (amplification control) [6]. In certain cases, absence of expected amplification including those for internal controls should be considered as an invalid reaction. In such cases, test should be repeated. If either of the target genes shows amplification, the result is considered as inconclusive. In such cases, RT-PCR test should be repeated before asking for repeat samples as many times obtaining repeat sample is difficult. This also minimizes the inconvenience to suffering patients and reduces the turn-around time for diagnosis. Repeat sample should be requested only if the similar results are obtained in case of both inconclusive and invalid samples, upon repeating the experiment.

\section{Sample collection and transport: issues with integrity and quality of target}

Proper collection of specimens is a vital step in the laboratory diagnosis of infectious diseases. Improper collection of specimens may lead to false negative test results. SARS$\mathrm{CoV} 2$ is present in different sites in an individual. The specimens most commonly preferred from upper respiratory tract are nasopharyngeal and/or oropharyngeal swabs. Nasopharyngeal swab is the reference sampling method to detect SARS-CoV-2, as recommended by the World Health Organization [15]. Samples collected from lower respiratory tract most commonly are bronchoalveolar lavage (BAL), tracheal aspirate and sputum $[14,15]$. As compared to $\mathrm{BAL}$, which requires special technical expertise and special tools, samples like saliva, sputum, nasal swab and throat swab are simple, safe and less painful. Other specimens like stool and blood can also be tested based on the necessity and stage of the disease progression. Additionally, for dead patients, autopsy material, lung tissues also can be examined. Samples should be collected in viral transport medium (VTMs). Once the sample is collected, it should be transferred immediately to the laboratory and the 
cold chain should be maintained during transport. In case of expected delays, specimen can be stored at $2-8{ }^{\circ} \mathrm{C}$ for up to $72 \mathrm{~h}$ after collection [4]. If further delays are expected, samples should be frozen to $-20{ }^{\circ} \mathrm{C}$ or ideally $-70{ }^{\circ} \mathrm{C}$ and shipped on dry ice [15]. It is important to avoid repeated freezing and thawing of specimens. Extracted RNA should be stored at $-20{ }^{\circ} \mathrm{C}$ for retesting if required. Failing to follow the above can lead to experimental failure or altered outcomes. Degradation of RNA due to improper transport or storage, may lead to false negative/inconclusive results with increased cycle threshold $(\mathrm{Ct})$ values. In negative controls, a fluorescence amplification curve obtained above the threshold level may indicate contamination. The absence of amplification in positive control indicates experimental failure. In all such cases, repeat experiments should be carried out.

\section{Conclusion}

In our opinion, the RT-PCR test results should be carefully interpreted. Detailed personal, family and medical history (Hypertension, Diabetes Mellitus, Asthma, Heart disease, Nephropathy, immune compromised individuals etc.) along with epidemiological factors are of paramount importance in interpreting the test results $[11,12,15]$. Negative RTPCR test results in cases with significant findings or history should be released with specific comments including the possibility of false negative results which may be on account of collection site, sample type and disease stage and in such cases repeat testing may be advisable. In case of inconclusive results, samples should be retested. For samples with repeated inconclusive results, repeat sample collection should be advised. Appropriate sampling procedures, standard laboratory protocols, stringent quality control norms, good quality RNA extraction and PCR kits can help in improving the accuracy of the test results.

Acknowledgements We acknowledge the encouragement and support from Director General, Indian Council of Medical Research (ICMR), Ministry for Health and Family Welfare, Government of India

Funding Not applicable.

\section{Declarations}

Conflict of interest All authors declare that they have no conflict of interest.

\section{References}

1. Alagarasu K, Choudhary ML, Lole KS, Abraham P, Potdar V, NIC Team. Evaluation of RdRp and ORF-1b-nsp14-based realtime RT-PCR assays for confirmation of SARS-CoV-2 infection: an observational study. Indian J Med Res. 2020;151(5):483-5. https://doi.org/10.4103/ijmr.IJMR_1256_20.

2. Bustin SA, Nolan T. RT-qPCR testing of SARS-CoV-2: a primer. Int J Mol Sci. 2020;21(8):3004. https://doi.org/10.3390/ ijms21083004.

3. Carter LJ, Garner LV, Smoot JW, Li Y, Zhou Q, Saveson CJ, Sasso JM, Gregg AC, Soares DJ, Beskid TR, Jervey SR, Liu C. Assay techniques and test development for COVID-19 diagnosis. ACS Cent Sci. 2020;6(5):591-605. https://doi.org/10.1021/acs centsci.0c00501.

4. Centers for Disease Control and Prevention (CDCP). Interim guidelines for collecting, handling, and testing clinical specimens for COVID-19. https://www.cdc.gov/coronavirus/2019-ncov/lab/ lab-biosafety-guidelines.html.

5. Chen LD, Li H, Ye YM, Wu Z, Huang YP, Zhang WL, Lin L. A COVID-19 patient with multiple negative results for PCR assays outside Wuhan, China: a case report. BMC Infect Dis. 2020;20(1):517. https://doi.org/10.1186/s12879-020-05245-7.

6. Dreier J, Störmer M, Kleesiek K. Use of bacteriophage MS2 as an internal control in viral reverse transcription-PCR assays. J Clin Microbiol. 2005;43(9):4551-7. https://doi.org/10.1128/JCM.43.9. 4551-4557.2005.

7. Goudouris ES. Laboratory diagnosis of COVID-19. J Pediatr (Rio J). 2021;97(1):7-12. https://doi.org/10.1016/j.jped.2020.08.001.

8. Khan KA, Cheung P. Presence of mismatches between diagnostic PCR assays and coronavirus SARS-CoV-2 genome. R Soc Open Sci. 2020;7(6):200636. https://doi.org/10.1098/rsos.200636.

9. Phan T. Genetic diversity and evolution of SARS-CoV-2. Infect Genet Evol. 2020;81:104260. https://doi.org/10.1016/j.meegid. 2020.104260.

10. Shen Z, Xiao Y, Kang L, Ma W, Shi L, Zhang L, Zhou Z, Yang J, Zhong J, Yang D, Guo L, Zhang G, Li H, Xu Y, Chen M, Gao Z, Wang J, Ren L, Li M. Genomic diversity of severe acute respiratory syndrome-coronavirus 2 in patients with coronavirus disease 2019. Clin Infect Dis. 2020;71(15):713-20. https://doi.org/ 10.1093/cid/ciaa203.

11. Tahamtan A, Ardebili A. Real-time RT-PCR in COVID-19 detection: issues affecting the results. Expert Rev Mol Diagn. 2020;20(5):453-4. https://doi.org/10.1080/14737159.2020. 1757437.

12. Wang Y, Kang H, Liu X, Tong Z. Combination of RT-qPCR testing and clinical features for diagnosis of COVID-19 facilitates management of SARS-CoV-2 outbreak. J Med Virol. 2020;92(6):538-9. https://doi.org/10.1002/jmv.25721.

13. Watson J, Whiting PF, Brush JE. Interpreting a COVID-19 test result. BMJ. 2020;369:m1808.

14. Wiseman J, D'Amico TA, Zawadzka S, Anyimadu H. False negative SARS-CoV-2 PCR-a case report and literature review. Respir Med Case Rep. 2020;31:101140. https://doi.org/10.1016/j. rmcr.2020.101140.

15. World Health Organization (WHO). Laboratory testing for coronavirus disease (COVID-19) in suspected human cases Interim guidance 19 March 2020. https://www.who.int/publica tions/i/item/10665-331501.

Publisher's Note Springer Nature remains neutral with regard to jurisdictional claims in published maps and institutional affiliations. 